\title{
Berichterstattung, Emotionen und politische Legitimierung
}

Eine experimentelle Untersuchung zum Einfluss der Politikberichterstattung auf die Legitimierung politischer Entscheidungen am Beispiel von Bundeswehreinsätzen

\section{Bertram Scheufele / Carolin Gasteiger}

Ob Bürger politische Entscheidungen legitimieren, hängt auch von der Mediendarstellung ab. Das wird exemplarisch für Auslandseinsätze der Bundeswebr untersucht. Die theoretischen Überlegungen wurden in einem Experiment geprüft. Der erste Faktor war der Bezugsrabmen: Die eine Version des Stimulusartikels stellte einen Krieg und Bundeswebreinsatz in einen humanitären Rabmen, die andere Artikelversion argumentierte politisch-militärisch. Der zweite Faktor manipulierte das Artikelfoto (Kinder vs. Kämpfer). Die humanitäre Rabmung rief stärkere Betroffenheit und Empathie hervor und fübrte zu größerer Zustimmung zum Bundeswehreinsatz als die politisch-militärische Darstellung. Das Foto kriegsleidender Kinder wirkte teilweise verstärkend. Daneben fungierten themenrelevante Einstellungen als Drittvariablen.

Schlagwörter: Medienwirkung, Politische Kommunikation, Legitimierung, Framing, Emotion, Bild

„Politik [ist...] ein überwiegend massenmedial ,vermitteltes' Geschehen“ (Sarcinelli 1998b: 702). Damit hängt von der Politikvermittlung der Medien in hohem Maße ab, ob politische Entscheidungen legitim erscheinen und Bürger sie befürworten oder ablehnen. Der vorliegende Beitrag fragt nicht danach, inwiefern die Medien politische Entscheidungen als legitim darstellten, sondern danach, inwiefern spezifische Merkmale der Politikberichterstattung beeinflussen, ob Bürger politische Entscheidungen legitimieren, $d$. h. als anerkennungswürdig beurteilen. Politikberichterstattung ist eine Funktion der politischen Realität und ihrer medialen Konstruktion (vgl. z. B. Bentele 1992; Kepplinger 1998, 2001). Wir können hier nicht klären, welchen Anteil die politische Realität an der medialen Darstellung politischer Entscheidungen hat. Vielmehr interessiert uns, ob signifikante Merkmale der Politikberichterstattung das Potenzial haben, die Legitimierung politischer Entscheidungen durch die Bürger zu beeinflussen.

\section{Politische Legitimität und Massenmedien}

Der Begriff politische Legitimität (vgl. z. B. Fuchs 1987; Greiffenhagen 1997; Westle 1989, 1990) verweist darauf, dass Regierende zwar „über ein gewisses Maß an Entscheidungsfreiheit [...] verfügen“ (Heller 1983: 247), sich aber stets gegenüber dem Volkssouverän rechtfertigen müssen (vgl. auch Tenscher 2003). ${ }^{1}$ Politische Herrschaft ist dabei sowohl begründungspflichtig als auch zustimmungsabhängig. „Beide, Zustimmung und

1 Den Begriff verwenden wir im Sinne Webers (1972), der u.a. drei Formen politischer Herrschaft bzw. Legitimierung benennt: traditionale, charismatische und rational-legale Herrschaft. Jene Position, die auf historische Formen legaler, aber nicht legitimer Herrschaft verweist (vgl. z. B. Westle 1989: 24f.) fordert dagegen festzulegen, worauf sich Legitimität beziehe (z. B. auf Werte wie Frieden, Freiheit, Solidarität). Während der Begriff ,Legitimierung' den prozessualen Aspekt reflektiert, markiert ,Legitimität' den Ergebnisaspekt. 
Begründung, finden ihre Realisierung durch und im Rahmen politischer Kommunikation" (Sarcinelli 1998a: 253). Diese erfolgt im intermediären System ,Politischer Öffentlichkeit', in dem auch die Massenmedien anzusiedeln sind. ${ }^{2} \mathrm{Zu}$ unterscheiden sind dabei zwei Richtungen (vgl. Jarren/Donges 2002a: 138ff.): Vom politisch-administrativen System zur Gesellschaft vollzieht sich Entscheidungsvermittlung: Politiker müssen z. B. ihre Entscheidung über die Entsendung von Bundeswehreinheiten gegenüber den Bürgern rechtfertigen (Begründungsaspekt). Das dürfte in vielen Fällen über die Medienberichterstattung laufen. In umgekehrter Richtung erfolgt Interessenvermittlung: So können Bürger z. B. ihre Ablehnung des G8-Gipfels in Demonstrationen kundtun oder Politiker bei der nächsten Wahl für Sicherheitsmaßnahmen wie in Heiligendamm abstrafen (Zustimmungsaspekt).

Politikwissenschaftliche Überlegungen zu Legitimität greifen neben Weber (1972) meist auf Eastons $(1975,1979)$ Konzept politischer Unterstützung zurück. Es benennt zum einen drei Bezugsobjekte für politische Unterstützung: die politische Gemeinschaft, die politische Ordnung und die amtierenden Herrschaftsträger. Zudem differenziert es diffuse und spezifische Arten politischer Unterstützung. Spezifische Unterstützung bezieht sich auf die Zufriedenheit der Bürger mit konkreten politischen Outputs (z. B. Gesetzen) bzw. Leistungen der Herrschaftsträger. Von Legitimierung spricht Easton $(1975,1979)$ nur bei einer breiten diffusen Unterstützung der Gesamtbevölkerung für das politische System ganz generell (vgl. Jacobs 1999: 11). Allerdings kann man annehmen, dass die Akzeptanz bzw. Anerkennung konkreter Outputs auch Auswirkungen auf die generelle Unterstützung für das politische System bzw. die demokratische Grundordnung hat.

Der vorliegende Beitrag beschäftigt sich mit spezifischen Outputs, d. h. mit der Legitimierung konkreter politischer Entscheidungen durch die Bürger. Wir fragen, welche Merkmale der Politikberichterstattung beeinflussen können, dass Bürger eine Entscheidung anerkennen und befürworten. Dabei setzen wir voraus, dass die Vermittlung politischer Entscheidungen und Begründungen in westlichen Demokratien heutzutage oftmals massenmedial erfolgt (vgl. als Überblick Jarren/Donges 2002a, b). Das gilt vor allem für Politikfelder außerhalb des direkten Erfahrungshorizonts der meisten Bürger - wie z. B. Außen- oder Sicherheitspolitik.

Medien spielen dabei eine zweifache Rolle (vgl. auch Sarcinelli 1998b; Scheufele 2005a; Tenscher 2003): Einerseits berichten sie über das politische Geschehen (z. B. die Verabschiedung eines Gesetzes) und transportieren dabei auch Begründungen der Politiker für Entscheidungen (vermittelnde Rolle). Andererseits stellen Medien das politische Geschehen auf spezifische Weise dar, indem sie z. B. Entscheidungen aus einer eigenen Perspektive diskutieren (prägende Rolle). Diese Rolle hängt aber von den Phasen im politischen Entscheidungsprozess ab (vgl. Jarren et al. 1996): Während die Medien bei der Problemartikulation noch als Filter und Verstärker neuer Themen fungieren und die Problemdefinition mit prägen, sind Medieneinflüsse in den Phasen der Politikdefinition, Programmentwicklung und Politikimplementierung relativ gering. ${ }^{3}$

2 „Intermediäre Systeme verbinden (mindestens) zwei externe Systeme, zwischen denen Kommunikationsschranken existieren" (Rucht 1991: 5).

3 Ausgehend von Phasenmodellen politischer Entscheidungen sind Medieneinflüsse auf die Legitimierung politischer Entscheidungen durch die Bürger im Vorfeld und Nachgang von Entscheidungen denkbar: Im Vorfeld bemühen Entscheidungsträger z. B. Bezugsrahmen, um eine parlamentarische Mehrheit zustande zu bringen und die Bürger auf die Entscheidung einzustimmen. Geben Medien diesen Bezugsrahmen ein Forum, dürfte die Zustimmung der Bürger 


\section{Effekte medialer Rahmungen und Nachrichtenbilder auf die Legitimierung politischer Entscheidungen - am Beispiel von Bundeswehreinsätzen}

Die Zustimmung der Bürger für politische Entscheidungen dürfte von der Medienberichterstattung mit beeinflusst werden. Dabei spielen verschiedene Merkmale der Berichterstattung eine Rolle (vgl. z. B. Scheufele 2005a). So können Medien z. B. den Argumenten für eine bestimmte Position ein Forum bieten und damit diese Position direkt stützen (vgl. Kepplinger et al. 1989). Daneben sind aber auch indirekte, subtilere Formen medialer Legitimierung zu diskutieren. So können Medien z. B. den Ansprüchen eines Politikers auf ein hohes Parteiamt die Legitimität entziehen, indem sie dessen pikantes Privatleben ans Licht der Öffentlichkeit bringen (vgl. Scheufele 2007). Oder politische Entscheidungen können dadurch legitimiert werden, dass Medien die zu lösenden Probleme in bestimmte Bezugsrahmen stellen. Darauf fokussiert dieser Beitrag.

\subsection{Effekte medialer Bezugsrabmen}

Inwiefern mediale Rahmungen die Zustimmung bzw. Ablehnung der Bürger für eine politische Entscheidung z. B. zu neuen Sozialreformen oder zu einem Bundeswehreinsatz beeinflussen, lässt sich mit dem Framing-Konzept erklären (vgl. Scheufele 2003, 2004). Aus der Wirkungsperspektive sind vier Effekte zu unterscheiden: Medien-Frames können bereits bestehende Schemata von Rezipienten aktivieren (vgl. Price et al. 1997). Diese bleiben dann für einige Zeit aktiviert, was ihre spätere Aktualisierung erleichtert (Priming-Effekt). Kumulatives und konsonantes Framing kann die Vorstellungen der Rezipienten aber auch in Richtung des Medien-Frames verschieben (Transformationseffekt), aber auch erst ein Schema herausbilden (Etablierungseffekt). Relevanter für die Legitimitätsfrage sind Einstellungseffekte (vgl. Nelson et al. 1997): Berichten Medien z. B. wiederholt über Menschenrechtsverletzungen in einer Kriegsregion, dann machen sie den Humanitätsgedanken im Bewusstsein der Rezipienten salienter als andere Aspekte (kognitive Komponente). Dadurch schlägt sich die mit Humanität verbundene Bewertung (affektive Komponente) in der Einstellung zu einem Bundeswehreinsatz in der betreffenden Krisenregion nieder, die dann insgesamt positiver wird. Rezipienten dürften also der Entscheidung über einen solchen Bundeswehreinsatz häufiger zustimmen, wenn Medien den Konflikt bzw. den Bundeswehreinsatz im Krisengebiet humanitär rabmen statt ihn politisch-militärisch einzuordnen. Solche Effekte unterscheiden sich darin von Persuasionseffekten (vgl. als Überblick z. B. Schenk 2002), dass persuasive Botschaften neue Informationen vermitteln und damit Einstellungsveränderungen bewirken, während Medien-Frames bereits verfügbare Informationen salienter machen und über den beschriebenen Mechanismus Einstellungseffekte hervorrufen.

Neben Einstellungseffekten sind auch emotionale Framing-Effekte wahrscheinlich. Emotionen hat die Framing-Forschung bislang weitgehend vernachlässigt. In der Rezeptionsforschung werden sie dagegen seit Jahren umfangreich diskutiert (vgl. für einen Überblick z. B. die Beiträge in Wirth et al. 2007). Neuere Rezeptionsstudien (z. B. Mangold et al. 2001; Früh/Fahr 2006) rekurrieren dabei oft auf Appraisal-Ansätze (z. B. Scherer 1998, 2001; Ulich/Mayring 2003). Danach werden Emotionen durch sequen-

im Vorfeld der Entscheidung wachsen. Legitimierung ist jedoch keine endgültige Angelegenheit. Selbst wenn z. B. ein Bundeswehreinsatz eine breite Zustimmung im Parlament und bei den Bürgern findet, kann diese in Ablehnung umschlagen, wenn der Einsatz unerwartet viele Soldaten das Leben kostet. 
tielle Appraisal- bzw. Bewertungsschritte (z. B. in Bezug auf Relevanz, Folgen oder Normverträglichkeit) angestoßen. Das führt zu spezifischen Reaktionsmustern, die Handlungstendenzen, Gefühle, motorischen Ausdruck und physiologische Reaktionen umfassen. Allerdings sind exakte Prognosen, welcher Stimulus welche spezifische Emotionen auslöst, schwer möglich. Darüber hinaus beschäftigen sich Appraisal-Ansätze eher mit individualzentrierten Emotionen (vgl. ähnlich Manstead \& Fischer 2001: 224) und seltener mit Emotionen, die auf das soziale Umfeld bzw. andere Personen zielen. Gerade für politisch-gesellschaftliche Themen sind aber auch, soziale Emotionen zu berücksichtigen. ${ }^{4}$ Damit meinen wir nicht Emotionen im Rahmen von „social appraisal“ (z. B. Manstead \& Fischer 2001) oder „emotional contagion“ (z. B. Hatfield et al. 1994), sondern Emotionen, die sich aus Theorien prosozialen Verhaltens ableiten lassen (vgl. dazu Bierhoff \& Montada 1988) - also z. B. Mitleid oder Empathie. Solche Gefühle sind wahrscheinlich bei Themen wie Armut, Kriegen oder Naturkatastrophen. So dürfte die mediale Rahmung eines Krieges als humanitäre Notlage z. B. nicht nur Gefühle wie Betroffenheit, Trauer oder Schrecken auslösen, sondern vor allem auch Mitleid und Solidarität. In diese Richtung weisen Befunde zu Empathie durch informierende Mediendarstellungen. Laut Zillmann (2004) hatten z. B. die Bilder verhungernder Kinder die Zustimmung der amerikanischen Öffentlichkeit zur Intervention in Somalia begünstigt. Später hatten die Bilder herumgeschleifter toter US-Soldaten die Öffentlichkeit gegen den Somalia-Einsatz mobilisiert. Allerdings erklärt Zillmann den Nexus zwischen Emotion und Legitimierung nicht näher.

\section{Abbildung 1: Konsistenz-Triade zwischen Grundwerten, Emotionen und politischer Entscheidung}

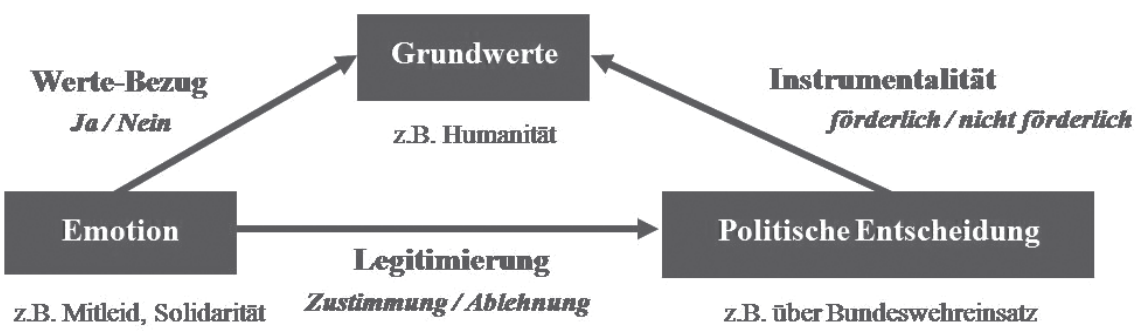

Nach Rosenbergs (1956) Theorie kognitiv-affektiver Konsistenz haben Menschen eine positive Einstellung zu einem Objekt, wenn es instrumentell nützlich für zentrale Werte ist. Je mehr solcher positiver Kognitionen, desto positiver ist die Gesamteinstellung zum Objekt - im Sinne kognitiv-affektiver Konsistenz. Unsere Argumentation knüpft daran an (Abbildung 1). Dazu folgendes Beispiel: Die humanitäre Rahmung eines Konfliktes, in dem die Bundeswehr intervenieren soll, ruft Mitleid und Solidarität hervor. Solche Emotionen ,appellieren' an Werte wie Menschenrechte oder Humanität (WerteBezug). ${ }^{5}$ Mit einer humanitären Rahmung legen Medien zugleich nahe, dass der Einsatz

4 Ortony et al. (1988) unterscheiden ereignis-, handlungs- und objektfundierte Emotionen und führen dabei auch Mitleid und Zuneigung als Beispiele an.

5 Mit dem Bezug zu Grundwerten lösen wir im Übrigen auch die von einigen Autoren (vgl. z. B. Westle 1989) geforderten Maßstäbe für politische Legitimität ein. 
für diese Werte förderlich ist - es sei denn sie behaupten explizit das Gegenteil (Instrumentalität). Daher dürften Rezipienten der Entscheidung über den Bundeswehreinsatz zustimmen (Legitimierung) - vor allem dann, wenn er auch noch explizit humanitär begründet wird. Rahmen Medien den Konflikt als politische Auseinandersetzung zwischen Regierung und Rebellen und einen Bundeswehreinsatz als strategisch-politische Intervention, dann fehlt positive Instrumentalität in Bezug auf Humanität, was in einer schwächeren Legitimierung münden dürfte. Stellen Medien einen Bundeswehreinsatz als menschenrechtswidrig dar und berichten über Opfer unter der Zivilbevölkerung, dann würde eine Ablehnung des Einsatzes aufgrund negativer Instrumentalität für Humanität begünstigt.

Einstellungsobjekte könnten durchaus für unterschiedliche Werte instrumentell nützlich sind. Ein Bundeswehreinsatz kann z. B. auch der internationalen Sicherheit dienen. Die entscheidende Frage ist, welche Werte höherrangig sind. Das ist je nach Einzelfall zu beurteilen bzw. zu prüfen. Bei Bundeswehreinsätzen dürfte der Normalbürger den für ihn greifbaren, konkreten Wert der Humanität und Menschlichkeit höherrangig einstufen als die eher abstrakte Wertkategorie internationaler Sicherheit. Bei nationalen Problemen kann das genau umgekehrt sein: Bei einer Zunahme von Kriminalität in der eigenen Region dürften sich viele Bürger im Zweifelsfall zugunsten von Sicherheit für sich selbst aussprechen statt für einen humanen Strafvollzug für die Täter.

Der Einstellungseffekt des Framing-Ansatzes und die beschriebene Rolle von Emotionen greifen im Ergebnis vermutlich ineinander, sind aber analytisch zu trennen. Um im Beispiel zu bleiben: Im ersten Fall macht die humanitäre Rahmung eines Krieges und Bundeswehreinsatzes zunächst den Aspekt der Humanität kognitiv salienter und erhöht damit auch dessen affektiven Stellenwert in der Einstellung zum Einsatz. Im zweiten Fall geht es nicht nur um positive bzw. negative Bewertungen bzw. Affekte, sondern um die ,Einordnung' von Affekten als spezifische Emotion (z. B. Empathie) im Sinne der beschriebenen Konsistenz-Verhältnisse.

\subsection{Effekte korrespondierender Nachrichtenbilder}

Neben der medialen Rahmung politischer Probleme bzw. Entscheidungen dürften auch visuelle Darstellungen - etwa Bilder von Armut - die Legitimierung einer politischen Entscheidung durch die Bürger beeinflussen. So zeigen etwa aufmerksamkeitstheoretische Arbeiten (z. B. Sargent/Zillmann 1999), dass dramatische Bilder die Überschätzung von Problemen und Gefahren begünstigen. Interessant sind aber vor allem Bilder, die sich mit der medialen Rahmung im Zeitungsartikel oder Sprechertext in einem Nachrichtenbeitrag decken. Damit geht es um die Frage von Text-Bild-Redundanz: Bilder fördern die Informationsaufnahme, wenn sie textredundant sind (vgl. als Überblick Brosius 1995). Scheufele (2001) hat das auf Framing übertragen: Bilder können mit dem verbalen Bezugsrahmen korrespondieren und ihn unterstützen. Das wäre z. B. der Fall, wenn ein Artikel einen Bundeswehreinsatz aus einer humanitären Perspektive diskutiert und das Foto kriegsleidender Kinder zeigt. Eine Text-Bild-Schere würde dagegen bei einem Foto bewaffneter Kämpfer vorliegen.

Zwar beschränken sich die meisten Studien zur Text-Bild-Redundanz auf die Erinnerungsleistung der Rezipienten. Das schließt jedoch Effekte auf Emotionen oder Meinungen nicht aus. So führte z. B. ein Zeitungsbericht über Zecken als Krankheitsüberträger dazu, dass die Rezipienten eine angebliche neue Krankheit für gefährlicher hielten, wenn der Artikel mit dem Foto einer Zecke illustriert war (vgl. Sargent/Zillmann 1999). Vergleichbares ist für unsere Fragestellung anzunehmen: Wenn das Foto 
mit dem inhaltlichen Bezugsrahmen korrespondiert, dürften die postulierten Effekte verstärkt werden. Das betrifft vermutlich vor allem die emotionalen Wirkungen: So dürfte ein Foto, das kriegsleidende Kinder zeigt, direkter als der Bezugsrahmen einer humanitären Nothilfe zu Emotionen wie Betroffenheit und Empathie führen. Denn hier entfalten „biologisch vorprogrammierte und kulturübergreifende“ (Kroeber-Riel 1996: 171) Muster ihre Wirkung, was auch in anderen Kontexten erkennbar wurde (vgl. z. B. Scheufele 2005b).

Die postulierten Wirkungszusammenhänge können durch Drittvariablen moderiert werden. Dazu gehören z. B. das Politikinteresse der Bürger und themenspezifische Einstellungen (vgl. als Überblick Scheufele 2004), aber auch Politikverdrossenheit (vgl. Wolling 1999; Maurer 2003): Wer mit dem politischen System unzufrieden ist und kein Vertrauen in politische Institutionen hat, dürfte auch selten den spezifischen Output befürworten.

\subsection{Anwendungsbeispiel, Bundeswehreinsätze}

Die theoretisch erörterten Effekte medialer Rahmung und korrespondierender Nachrichtenbilder auf die Legitimierung politischer Entscheidungen durch die Bürger lassen sich nur an einem konkreten Problemfall empirisch überprüfen. Wir haben uns aus vier Gründen für das Thema, Auslandseinsätze der Bundeswebr ${ }^{c}$ entschieden: Erstens liegen Bundeswehreinsätze außerhalb des Erfahrungsbereichs der meisten Menschen, was Medieneinflüsse wahrscheinlicher macht als bei Routinethemen im direkten Erfahrungshorizont. Zweitens erfordert die Entsendung von Bundeswehreinheiten eine breite parteiübergreifende Mehrheit im Bundestag. Damit dürften auch die Parteipräferenzen der Rezipienten eine geringere Rolle spielen als bei anderen Themen.

Drittens lassen sich am Beispiel der Bezugsrahmen im politischen und medialen Diskurs über Auslandseinsätze unsere theoretischen Überlegungen besonders gut überprüfen. Zu unterscheiden sind dabei folgende Diskurslinien und Bezugsrabmen: In verfassungsrechtlicher Hinsicht darf die Bundeswehr an keinem Angriffskrieg teilnehmen (Art. 26 GG), da ihre primäre Aufgabe die Landesverteidigung ist (Art. 87a GG). In völkerrechtlicher Hinsicht werden das Gewaltverbot der UN-Charta und das Prinzip der Nicht-Intervention wirksam. Stellt der Sicherheitsrat nach Art. 39 UN-Charta eine Friedensgefährdung fest und beschließt nach Art. 42 den Einsatz militärischer Sanktionen, dann legitimiert ein UN-Mandat zu Waffengewalt (vgl. Steinkamm 2000). Ohne UN-Mandat sind Auslandseinsätze der Bundeswehr nicht verfassungskonform. Eine Ausnahme ist der Bündnisfall durch die NATO-Mitgliedschaft (vgl. Art. 24, Abs. 2 GG). Ohne UN-Mandat, aber im Rahmen der NATO-Operation und mit dem Argument humanitärer Nothilfe nahmen Bundeswehrsoldaten erstmals an einem Out-ofArea-Einsatz im Kosovo 1999 teil. Schwab-Trapp (2002: 388) fasst die Entwicklung der Diskurslinien so zusammen: „Das [...] die politische Praxis militärischer Selbstbeschränkung begründende Gebot ,Nie wieder Krieg', das die deutsche politische Kultur seit 1945 geprägt hat, ist dem Imperativ gewichen, gegen massive Menschenrechtsverletzungen auch mit militärischen Mitteln vorzugehen“".

Mit dieser Skizze lässt sich dem im letzten Jahrzehnt immer bedeutsamer gewordenen Bezugsrahmen der bumanitären Nothilfe eine rein politisch-militärische Argumentation gegenüberstellen (vgl. Eilders/Lüter 2000; Robinson 2000a, b; Scheufele 2005a). Im ersten Fall wird mit Menschenrechtsverletzungen und der Notlage der Bevölkerung argumentiert, im zweiten Fall damit, dass Kriegshandlungen beendet und Konfliktparteien 
zum Einlenken bewegt werden (vgl. auch Iyengar/Simon 1993). ${ }^{6}$ Wie erwähnt, dürfte für den durchschnittlichen Bürger der humanitäre Grundwert schwerer wiegen als die Frage außenpolitischer Strategie oder internationaler Sicherheit. Darüber hinaus spielen Nachrichtenbilder in der Kriegsberichterstattung eine wichtige Rolle (vgl. z. B. Knieper/ Müller 2005). Die geläufigsten Bildmotive sind dabei Verwundete, Flüchtlinge, Mütter mit Kindern und bewaffnete Soldaten bzw. Rebellen (vgl. Reeb 2004). Damit ließen sich für die beiden Bezugsrahmen jeweils korrespondierende Nachrichtenbilder finden.

\section{Hypothesen}

Den Medieneinfluss auf die Zustimmung der Bürger zu politischen Entscheidungen untersuchten wir exemplarisch für Auslandseinsätze der Bundeswehr. Ausgehend vom tatsächlichen politischen und medialen Diskurs kontrastierten wir eine humanitäre mit einer rein politisch-militärischen Rahmung. Im Experiment lasen die Probanden einen Zeitungsartikel, der die Parlamentsentscheidung über den angeblichen Einsatz der Bundeswehr in einem fiktiven afrikanischen Land thematisierte. Mit den Befunden zur Empathie aufgrund informierender Mediendarstellungen war anzunehmen, dass die humanitäre Rahmung des Konfliktes, in dem die Bundeswehr angeblich interveniert, bei Probanden Betroffenheit (z. B. Trauer, Wut) und Empathie (z. B. Solidarität, Mitleid) auslöst. Das ist bei einer politisch-militärischen Rahmung unwahrscheinlicher. Daraus ergibt sich folgende Hypothese (Haupteffekt, Textlicher Bezugsrahmen'):

Hypothese 1a: Leser des Artikels mit humanitärem Rabmen zeigen häufiger Betroffenheit und Empathie als Leser des Artikels mit politisch-militärischem Rabmen.

Ausgehend von den in der Kriegsberichterstattung geläufigen Bildmotiven lassen sich Kinder als Kriegsopfer und Militärs/Rebellen als Kriegsakteure kontrastieren. Mit psychologischen Erkenntnissen dürften Fotos leidender Kinder eher Betroffenheit und Empathie auslösen als Fotos bewaffneter Krieger. Daraus ergibt sich folgende Hypothese (Haupteffekt ,Nachrichtenbild'):

Hypothese 1b: Leser des Artikels mit Foto der Kinder zeigen häufiger Betroffenheit und Empathie als Leser des Artikels mit Foto der Militärs.

Aufgrund empirischer Befunde zur Text-Bild-Redundanz ist anzunehmen, dass korrespondierende Nachrichtenbilder den emotionalen Effekt des Bezugsrahmens verstärken. Daraus ergibt sich folgende Hypothese (Interaktionseffekt ,Textlicher Bezugsrahmen und Nachrichtenbild'):

Hypothese 1c: Leser des Artikels mit humanitärem Rabmen und Foto der Kinder zeigen am häufigsten Betroffenheit und Empathie, Leser des Artikels mit politisch-militärischem Rabmen und Foto der Militärs am seltensten.

Die durch humanitäre Rahmung hervorgerufenen Emotionen appellieren an Werte wie Menschenrechte und Humanität (positiver Werte-Bezug). Der Bundeswehreinsatz erscheint bei humanitärer Rahmung als förderlich für diese Werte (positive Instrumentalität). Aus Konsistenzgründen dürften Rezipienten daher der Entscheidung über den Einsatz zustimmen (Abbildung 1). Bei politisch-militärischer Rahmung fehlt positive

6 Sicher sind auch andere Motive und Argumente denkbar: Während z. B. die Bush-Regierung den Irak-Krieg 2003 als „Befreiung Iraks“ darzustellen versuchte, wurden ihr von anderer Seite wirtschaftliche und strategische Motive unterstellt (vgl. Szukala 2003; Löffelholz 2004). 
Instrumentalität in Bezug auf Humanität, was in einer geringeren Legitimierung münden dürfte. ${ }^{7}$ Mediale Rahmung kann nach dem Framing-Ansatz aber auch direkt die Legitimierung der Rezipienten beeinflussen: Bei humanitärer Rahmung wird der $\mathrm{Hu}-$ manitätsaspekt salienter und überlagert andere Aspekte, deren affektive Bedeutung für die Gesamteinstellung sinkt. Beide Wirkungsmechanismen dürften im Ergebnis zusammenspielen. Daraus ergibt sich folgende Hypothese (Haupteffekt ,Textlicher Bezugsrahmen'):

Hypothese 2a: Leser des Artikels mit humanitärem Rabmen legitimieren den Bundeswebreinsatz bäufiger als Leser des Artikels mit politisch-militärischem Rabmen.

Die durch Nachrichtenfotos ausgelösten Emotionen dürften vergleichbare Effekte auf die Legitimierung haben. Daraus ergibt sich folgende Hypothese (Haupteffekt ,Nachrichtenbild'):

Hypothese 2b: Leser des Artikels mit Foto der Kinder legitimieren den Bundeswehreinsatz bäufiger als Leser des Artikels mit Foto der Militärs.

Schließlich ist anzunehmen, dass korrespondierende Nachrichtenbilder den Effekt des Bezugsrahmens auf die Legitimierung des Bundeswehreinsatzes verstärken. Daraus ergibt sich folgende Hypothese (Interaktionseffekt, Textlicher Bezugsrahmen und Nachrichtenbild'):

Hypothese 2c: Leser des Artikels mit humanitärem Rabmen und Foto der Kinder legitimieren den Bundeswehreinsatz am häufigsten, Leser des Artikels mit politisch-militärischem Rabmen und Foto der Militärs am seltensten.

Darüber hinaus hatten wir eine Reihe denkbarer Rezipientenvariablen diskutiert, die als Drittvariablen die postulierten Wirkungszusammenhänge verstärken bzw. abschwächen. Dazu gehören die politische Mediennutzung der Probanden, ihr Politikinteresse, vor allem aber ihre Einstellungen zur Bundeswehr und deutschen Außenpolitik sowie ihre generelle Politikverdrossenheit.

Forschungsfrage: Welche Rolle spielen politisch relevante Rezipientenvariablen für die postulierten Wirkungszusammenhänge?

\section{Untersuchungsanlage und Erhebungsinstrument}

Die Hypothesen wurden in einem zweifaktoriellen Experiment überprüft. Als Probanden nahmen 132 Studierende der Kommunikationswissenschaft aus fünf Einführungskursen im ersten Studiensemester teil. Ihnen wurde vermittelt, dass sie an einer Befragung zur Qualität der Politikberichterstattung teilnehmen würden. Die Geschlechter- und die Altersverteilung unterschieden sich nicht in den vier Experimentalbedingungen, sodass die Randomisierung als gelungen gelten kann. ${ }^{8}$ Die Probanden lasen einen Artikel über

7 Wie oben erläutert, kann die politisch-militärische Rahmung zwar ebenfalls an zentrale Werte wie etwa Sicherheit appellieren. Bei Bundeswehreinsätzen dürfte der Normalbürger den greifbaren Wert der Humanität aber höherrangig einstufen als die abstrakte Wertkategorie internationaler Sicherheit.

8 Der Anteil der weiblichen Versuchspersonen betrug in Gruppe 1 ( $n=34) 74 \%$, in Gruppe 2 $(n=31) 84 \%$, in Gruppe 3 ( $n=32) 75 \%$ und in Gruppe $4(n=35) 69 \%\left(\mathrm{Chi}^{2}=2,12 ; \mathrm{df}=3\right.$; n.s.). Das Durchschnittsalter lag in Gruppe 1 bei 20,4 Jahren (Std. Abw. 2,0), in Gruppe 2 bei 21,5 
die Parlamentsentscheidung zum angeblichen UN-Einsatz der Bundeswehr in Mlandi. In der Artikelmitte war ein Foto eingebettet. Der erste Experimentalfaktor war der Bezugsrahmen, in den der Artikel den Konflikt und die Entscheidung über den Bundeswehreinsatz stellte. Der zweite Faktor war das Nachrichtenbild (Tabelle 1). ${ }^{9}$

Tabelle 1: Experimentelles Design der Untersuchung (eigene Darstellung)

\begin{tabular}{llll}
\hline & & \multicolumn{2}{l}{ Inhaltlicher Bezugsrahmen im Zeitungsartikel } \\
& & Humanitäre & Politisch-militärische \\
& & Rahmung & Rahmung \\
\hline \multirow{3}{*}{ Nachrichtenbild } & \multirow{2}{*}{ Kinder als Opfer } & Text-Bild- & Text-Bild- \\
& \multirow{2}{*}{ Bewaffnete Rebellen } & Korrespondenz & Schere \\
& & Text-Bild- & Text-Bild- \\
& & Schere & Korrespondenz \\
\hline
\end{tabular}

Der Artikel berichtete über einen sich angeblich in Mlandi zuspitzenden Konflikt und über die Entscheidung der Bundesregierung, Bundeswehrsoldaten im Rahmen eines UN-Mandats zu entsenden. Die Gestaltung des Treatments orientierte sich an den Charakteristika des politischen und medialen Diskurses im betreffenden Politikfeld (vgl. Jertz, 2001; Meder 1998; Schwab-Trapp 2000, 2002; Robinson 2000a, b; Scheufele 2005a).

Manipuliert wurden Art und Ausmaß des Konflikts sowie die Argumente für die Entsendung der Bundeswehreinheiten. Das erfolgte weitgehend über Schlagworte und Formulierungen, sodass die beiden Artikelversionen ansonsten konstant waren. Die politisch-militärisch rahmende Artikelversion lenkte den Blick auf den Konflikt zwischen Rebellen und Regierungstruppen (z. B. „gehen die Kämpfe zwischen Rebellen und Regierung weiter"). Die humanitär rahmende Artikelversion betonte die humanitäre Notlage (z. B. „geht das Leiden für die Zivilbevölkerung weiter"). Der politisch-militärisch rahmende Artikel begründete den Einsatz der Bundeswehreinheiten damit, die Kriegshandlungen zu stoppen (z. B. „die politischen Kämpfe zu beenden“ oder „mangelnde Einigungsbereitschaft zwischen Rebellen und Regierung“), der andere Artikel betonte die humanitäre Nothilfe (z. B. „die humanitäre Katastrophe zu beenden“ oder „immer brutaleren Übergriffen der Rebellen auf die Zivilbevölkerung"). Die Artikelversionen enthielten z. B. auch Einschätzungen von Politikern zur Lage in Mlandi: Im politisch-militärischen Artikel sprachen Bundestagsabgeordnete von einer „politisch höchst brisanten Situation“, im humanitär argumentierenden Artikel von einer „menschenunwürdigen Situation“.

Das mit der politisch-militärischen Artikelversion korrespondierende Foto stammte von ,Spiegel Online.$^{6}{ }^{10}$ Im Original zeigt es vier sudanesische Rebellen, die ihre Gewehre

Jahren (Std. Abw. 3,6), in Gruppe 3 bei 21,8 Jahren (Std. Abw. 2,9) und in Gruppe 4 bei 20,8 Jahren (Std. Abw. 2,6) $(\mathrm{F}=1,56 ; \mathrm{df}=3$; n.s.).

9 Das „westafrikanische Land Mlandi“ und der dortige Konflikt waren frei erfunden, aber glaubwürdig. Denn fast alle der an einem Pretest teilnehmenden Personen waren der festen Überzeugung, das Land zu kennen. Der Landesname und weitere Eigennamen für die Konfliktparteien (Mako, Kimbi und Liberation Union) lehnten sich an Rabehls (2000) Erläuterungen zu afrikanischen Kriegen sowie an Löffelholz (2004) an.

10 http://www.spiegel.de/dossiers/ausland/0,1518,298628,00.html (Abruf: 18.10.2005). 
hochhalten, wobei einer im Vorder- und die anderen im Hintergrund stehen. Das zur humanitären Version redundante Foto stammte von der Homepage des Roten Kreuzes. ${ }^{11}$ Im Original zeigt es vier sudanesische Flüchtlinge: Ein kleiner Junge mit verbundenem Arm steht vor drei Familienmitgliedern. Die Fotos waren in Größe und Bildaufteilung vergleichbar. ${ }^{12}$

Der Fragebogen ermittelte im ersten Block die politische Mediennutzung, die Parteineigung und das politische Interesse der Probanden für neun Politikfelder - darunter Außenpolitik. Danach folgte das Treatment. Im zweiten Block wurden drei Pufferfragen zur Qualität des Zeitungsartikels gestellt. Danach wurden als erste abhängige Variable die Emotionen anhand unipolarer 5er-Skalen („Trifft überhaupt nicht zu“ bis „Trifft stark zu“) erfasst. Vorgegeben waren zehn allgemeine Emotionen aus der „Differentiellen Affekt-Skala" (Merten/Krause 1993) und vier ,soziale` Emotionen (Solidarität, Mitleid, Hass, Verachtung). Die aus Appraisal-Ansätzen abgeleiteten Emotionen waren z. B. „Ich bin betroffen“. Die sozialen Emotionen wurden eingeleitet mit „Ich verspüre ...". Die Emotionen wurden ohne Vorgabe eines Bezugsobjekts abgefragt, denn Menschen können allgemeine Emotionen bei komplexen Sachverhalten vermutlich nur bedingt Einzelbezügen zuschreiben. Die ,sozialen` Emotionen lassen sich wiederum vor dem Hintergrund des Treatments relativ leicht zuordnen: So dürften die meisten Menschen z. B. eher Mitleid mit zivilen Opfern als mit tötenden Soldaten haben.

Der dritte Fragenblock erfasste die zentrale abhängige Variable, also die Legitimierung des Bundeswehreinsatzes. Die Probanden sollten anhand unipolarer 5er-Skalen ihre Zustimmung zu zehn Aussagen ausdrücken. Diese bezogen sich auf Souveränität, Solidarität, internationales Engagement sowie politisch-militärische und humanitäre Dimensionen und repräsentierten verschiedene Grade der Zustimmung zum Einsatz. Die weiteren Fragenblöcke umfassten themenrelevante Drittvariablen. Da Auslandseinsätze der Bundeswehr eine breite Parlamentsmehrheit erfordern, dürfte die Parteineigung der Probanden bei diesem Thema weniger wirksam werden. Die Voreinstellungen zur Bundeswehr und zur deutschen Außen- und Sicherheitspolitik können dagegen eine verstärkende oder eine abschwächende Rolle spielen. Die Politikverdrossenbeit wurde mit einer Listenfrage erhoben. In Anlehnung an Wolling (1999) und Maurer (2003) sollten die Probanden anhand unipolarer 5er-Skalen ihre Zustimmung zu sieben Aussagen über politische Unterstützung und Partizipation ausdrücken. Die Einstellungen zur Bundeswehr erfassten wir mit zwei Fragen aus einer Umfrage zur Bundeswehr (vgl. AIK 2001): Die Probanden sollten ihre Haltung zur Bundeswehr anhand einer bipolaren 5er-Skala ausdrücken. Zudem sollten sie ihr Vertrauen in neun Institutionen angeben - darunter die Bundeswehr. In Anlehnung an Schwab-Trapp (2002) und die Bundeswehr-Umfrage (AIK 2001) haben wir auch die Haltung zur deutschen Außenpolitik erhoben. Dazu sollten die Probanden anhand unipolarer 5er-Skalen angeben, für wie wichtig sie sieben politische Aufgaben der Bundesrepublik erachten - darunter auch „Friedenssicherung notfalls mit Waffengewalt". Zuletzt wurden als soziodemographische Merkmale Alter und Geschlecht erhoben.

11 http://www.redcross.ch/aid/donations/spende-de.php?id=2\&h=i\&a=html (Abruf: 18.10.2005).

12 Der denkbare Einwand, dass sie sich in zwei Dimensionen - in Rolle (Rebellen, Zivilisten) und Alter (Erwachsene, Kinder) der Akteure - unterscheiden, lässt sich mit dem Argument entkräften, dass die spezifische Kombination beider Dimensionen der faktischen Mediendarstellung anderer Konflikte und Kriege entspricht. 


\section{Ergebnisse}

Nach Hypothese 1a sollte die humanitäre Rahmung des Artikels stärker Emotionen der Betroffenheit und Empathie bei den Probanden auslösen als die rein politisch-militärische Rahmung. Vergleichbares postulierte Hypothese $1 \mathrm{~b}$ für das Foto kriegsleidender Kinder im Vergleich zum Foto bewaffneter Krieger. Hypothese 1c unterstellte einen additiven Effekt beider Faktoren.

Tabelle 2: Faktorenanalyse für allgemeine Emotionen der ProbandenHauptkomponentenanalyse, Orthogonalrotation, Kaiserkriterium

\begin{tabular}{|c|c|c|c|}
\hline Faktor 1 „Wut“ & & & \\
\hline Ich bin wütend & 0.81 & & \\
\hline Ich bin verärgert & 0.76 & & \\
\hline Ich bin enttäuscht & 0.63 & & \\
\hline Ich bin handlungsbereit & 0.63 & & \\
\hline \multicolumn{4}{|l|}{ Faktor 2 „Schock“ } \\
\hline Ich bin erschrocken & & 0.79 & \\
\hline Ich bin traurig & & 0.75 & \\
\hline Ich bin betroffen & & 0.60 & \\
\hline \multicolumn{4}{|l|}{ Faktor 3 „Angst“ } \\
\hline Ich bin ängstlich & & & 0.75 \\
\hline Ich fühle mich entmutigt & & & 0.69 \\
\hline Ich fühle mich schuldig & & & 0.63 \\
\hline
\end{tabular}

Skala jeweils von 1 („trifft überhaupt nicht zu“) bis 5 („trifft stark zu“); $\mathrm{n=127}$ Probanden. Ausgewiesen sind Faktorladungen > 0.40; KMO=0.71; Erklärte Gesamtvarianz: 59 \%

Die zehn Emotionen aus der „Differentiellen Affekt-Skala“ haben wir zunächst einer Faktorenanalyse unterzogen. Bei einer erklärten Varianz von 59 Prozent legte die beste Lösung drei Faktoren nahe, die mit „Wut“, „Schock“ und „Angst“ etikettiert wurden (Tabelle 2). Zudem hatten wir vier ,soziale‘ Emotionen erfasst. Für die drei Emotionsfaktoren und die vier, sozialen' Emotionen ergaben zweifaktorielle Varianzanalysen fast durchweg signifikante Haupteffekte für den Bezugsrahmen, in den der Konflikt in Mlandi bzw. der Bundeswehreinsatz gestellt wurde: Hatten Probanden den Artikel gelesen, der den Konflikt und Einsatz humanitär rahmte, dann waren sie schockierter, aber weniger ängstlich als Leser des politisch-militärisch argumentierenden Artikels. Zudem hatten sie mehr Solidarität und Mitleid (wohl für die Kriegsopfer) und mehr Verachtung (wohl für die Kriegsakteure). Insgesamt zeigten die Probanden bei humanitärer Rahmung also ein emotionales Gesamtbild von Empathie und Solidarität, das sie bei politisch-militärischer Perspektive seltener erkennen ließen. Damit bestätigte sich Hypothese 1a. ${ }^{13}$ Das Foto spielte nur beim Faktor „Wut“ eine Rolle. Interaktionseffekte waren nicht festzustellen. (Tabelle 3)

13 Für „Hass“ ergaben sich weder signifikante Haupt- noch signifikante Interaktionseffekte. 
Tabelle 3: Allgemeine und, soziale`Emotionen der Probanden-Signifikante Haupteffekte für Bezugrabmen und Nachrichtenbild

\begin{tabular}{|c|c|c|c|c|c|}
\hline Allgemeine Emotionen & $\begin{array}{l}\text { Haupt-/ } \\
\text { Interaktionseffekt }\end{array}$ & $\mathrm{F}$ & $\mathrm{p}$ & Ausprägungen & $\mathrm{MW}^{\mathrm{A})}$ \\
\hline \multirow{2}{*}{ Faktor 1 „Wut“ } & \multirow{2}{*}{$\begin{array}{l}\text { Haupteffekt Faktor } 2 \\
\text { „Nachrichtenbild“ }\end{array}$} & \multirow{2}{*}{4,84} & \multirow{2}{*}{$<0.05$} & Kinder & +0.2 \\
\hline & & & & Krieger & -0.2 \\
\hline \multirow[b]{2}{*}{ Faktor 2 „Schock“ } & Haupteffekt Faktor 1 & \multirow[b]{2}{*}{12,21} & \multirow[b]{2}{*}{$<0.01$} & Humanitär & +0.3 \\
\hline & $\begin{array}{l}\text { „Textlicher } \\
\text { Bezugsrahmen“ }\end{array}$ & & & Politisch-militärisch & -0.3 \\
\hline \multirow[b]{2}{*}{ Faktor 3 „Angst“ } & Haupteffekt Faktor 1 & \multirow[b]{2}{*}{7,07} & \multirow[b]{2}{*}{$<0.01$} & Humanitär & -0.2 \\
\hline & $\begin{array}{l}\text { „Textlicher } \\
\text { Bezugsrahmen“ }\end{array}$ & & & Politisch-militärisch & +0.2 \\
\hline ,Soziale' Emotionen & $\begin{array}{l}\text { Haupt-I } \\
\text { Interaktionseffekt }\end{array}$ & $\mathrm{F}$ & $\mathrm{p}$ & Ausprägungen & $\mathrm{MW}^{\mathrm{B})}$ \\
\hline \multirow[b]{2}{*}{ Solidarität } & Haupteffekt Faktor 1 & \multirow[b]{2}{*}{7,03} & \multirow[b]{2}{*}{$<0.01$} & Humanitär & 3,8 \\
\hline & $\begin{array}{l}\text { „Textlicher } \\
\text { Bezugsrahmen“ }\end{array}$ & & & Politisch-militärisch & 3,3 \\
\hline \multirow[b]{2}{*}{ Mitleid } & Haupteffekt Faktor 1 & \multirow[b]{2}{*}{22,83} & \multirow[b]{2}{*}{$<0.01$} & Humanitär & 4,3 \\
\hline & $\begin{array}{l}\text { „Textlicher } \\
\text { Bezugsrahmen“ }\end{array}$ & & & Politisch-militärisch & 3,5 \\
\hline \multirow[b]{2}{*}{ Verachtung } & Haupteffekt Faktor 1 & \multirow[b]{2}{*}{7,65} & \multirow[b]{2}{*}{$<0.01$} & Humanitär & 2,5 \\
\hline & $\begin{array}{l}\text { „Textlicher } \\
\text { Bezugsrahmen“ }\end{array}$ & & & Politisch-militärisch & 2,0 \\
\hline
\end{tabular}

Ausgewiesen sind signifikante Haupteffekte. Interaktionseffekte traten nicht auf; $n=127$ Probanden

A) Faktorladungen

B) Skala jeweils von 1 („trifft überhaupt nicht zu“) bis 5 („trifft stark zu“)

Inhaltliche Rahmung und Nachrichtenbild sollten sich nach den Hypothesen 2a bis 2c auch auf die Legitimierung des Bundeswehreinsatzes durch die Probanden auswirken. Dafür können zwei Mechanismen wirksam werden (vgl. Abschnitt 2): Einerseits macht nach dem Framing-Konzept die humanitäre Rahmung den Aspekt der Humanität im Bewusstsein der Probanden salienter (kognitive Komponente), wodurch sich die daran geknüpfte positive Bewertung (affektive Komponente) stärker in der Gesamteinstellung zum konkreten Bundeswehreinsatz niederschlägt. Andererseits appellieren die hervorgerufenen Emotionen an Grundwerte wie Humanität oder Menschenrechte. Da die humanitäre Rahmung den Bundeswehreinsatz als förderlich für diese Grundwerte vermittelt, sollten Probanden aus Konsistenzgründen den Einsatz legitimieren. Vergleichbares ist bei einer rein politisch-militärischen Rahmung nicht zu erwarten.

Die Probanden sollten ihre Zustimmung zu zehn Aussagen angeben, die Befürwortung bzw. Ablehnung des Bundeswehreinsatzes repräsentierten. ${ }^{14}$ Die Aussagen wurden

14 Zustimmende Aussagen: „Ich finde es gut, dass sich Bundeswehrsoldaten in Mlandi für den Frieden einsetzen“, „Meiner Meinung nach sollten Bundeswehrsoldaten nach Mlandi geschickt werden, aber nur zur humanitären Hilfe" (aufgrund der Reliabilitätsanalyse von der Indexbildung ausgeschlossen), „Die internationale Gemeinschaft kann der Situation in Mlandi doch nicht einfach tatenlos zusehen. Ich bin dafür, Bundeswehrsoldaten nach Mlandi zu entsenden“, „Ich finde es gut, dass die deutsche Regierung mit dem Bundeswehreinsatz in Mlandi internationale Verantwortung übernimmt“ sowie „Bundeswehrsoldaten sollten nur für militärische 


\section{Abbildung 2: Legitimierung des Bundeswehreinsatzes durch die Probanden (Index) ${ }^{A)}$ - Zweifaktorielle Varianzanalysen}

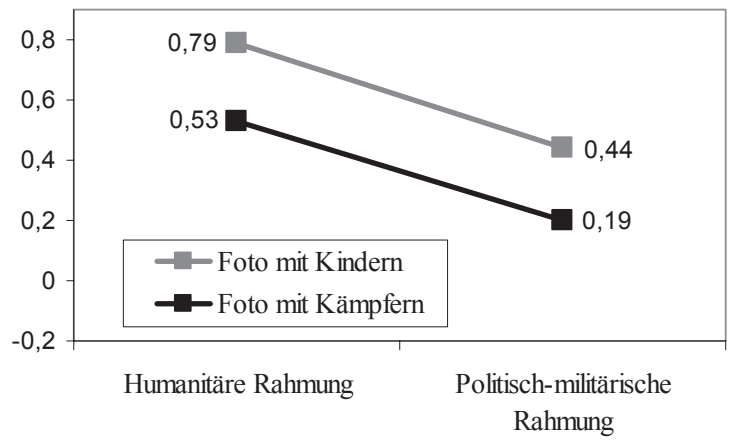

Haupteffekt Faktor 1 „Textlicher Bezugsrahmen" F=9,42, $\mathrm{p}<0.01$

Haupteffekt Faktor 2 „Nachrichtenbild“ $F=5,35$, $\mathrm{p}<0.05$

Interaktionseffekt $\mathrm{F}=0,00$, n.s.

$\mathrm{n}=130$ Probanden; $\mathrm{Eta}^{2}=0,11$

A) Die 10 Aussagen im Fragebogen wurden zu einem Legitimierungs-Index $I_{L}$ zusammengefasst; Werte von -2 („Starke Ablehnung“) bis +2 („Starke Zustimmung“): $I_{L}=\left(A_{1}+A_{2}+\ldots A_{n}\right) / n$. Die Reliabilitätsanalyse schloss zwei Aussagen von der Indexbildung aus (Chronbachs alpha $=0.80)$.

zu einem Index zusammengefasst, der Werte von -2 („Starke Ablehnung“) bis +2 („Starke Zustimmung") annehmen konnte; eine Reliabilitätsanalyse schloss zwei Aussagen von der Indexbildung aus (Chronbachs $\alpha=0.80$ ). Für die Legitimierung des Einsatzes zeigte sich ein signifikanter Haupteffekt sowohl für den Bezugsrahmen als auch das Nachrichtenfoto, was die Hypothesen $2 \mathrm{a}$ und $2 \mathrm{~b}$ bestätigte (Abbildung 2): Leser des Artikels mit humanitärer Rahmung stimmten dem Bundeswehreinsatz stärker zu (Legitimierungs-Index: $\mathrm{MW}=+0,7)$ als Leser des politisch-militärisch argumentierenden Artikels $(\mathrm{MW}=+0.3)$. Zudem sorgte das Foto kriegsleidender Kinder für größere $\mathrm{Zu}-$ stimmung $(\mathrm{MW}=+0.6)$ als das Foto bewaffneter Kriegsakteure $(\mathrm{MW}=+0.4) .{ }^{15}$

Es lässt sich nicht eindeutig klären, welcher der beiden Mechanismen - der emotionale oder der Einstellungseffekt - hier stärker wirksam wurde. Im Ergebnis greifen beide vermutlich ineinander. Gewisse Hinweise können wir aber aus den Korrelationen zwischen Emotionen und Legitimierung ziehen (Tabelle 4). Für das Aggregat aller Probanden zeigten sich signifikante Zusammenhänge: Die ,sozialen' Emotionen Solidarität und Mitleid korrelierten signifikant mit der Zustimmung zum Bundeswehreinsatz. Etwas schwächer waren die Korrelationen für die Emotionsfaktoren. Allerdings können die

Hilfe nach Mlandi entsendet werden“ (aufgrund der Reliabilitätsanalyse von der Indexbildung ausgeschlossen) - Ablehnende Aussagen: „Ich bin dagegen, dass bewaffnete Bundeswehrsoldaten nach Mlandi geschickt werden“, „Ich bin dagegen, dass Bundeswehrsoldaten aus machtpolitischen Gründen nach Mlandi entsendet werden sollen“, „Bundeswehrsoldaten sollten nie nach Mlandi entsendet werden“, „Rebellen und Regierungstruppen sollten ihre Streitigkeiten in Mlandi untereinander lösen“ sowie „Die deutsche Regierung mischt sich in Mlandi zu sehr in ausländische Angelegenheiten ein“.

15 Die angegebenen Werte sind Mittelwerte für den jeweiligen Haupteffekt. Sie sind nicht identisch mit den Mittelwerten für die Faktorkombination in Abbildung 3. 
Tabelle 4: Zusammenbänge zwischen Legitimierung und Emotionen der Probanden - Pearson-Korrelationskoeffizient

\begin{tabular}{|c|c|c|c|c|c|}
\hline \multicolumn{6}{|l|}{ Alle Probanden } \\
\hline Legitimierung und ... & Pearson-Korr. & $\mathrm{p}$ & Legitimierung und ... & Pearson-Korr. & $\mathrm{p}$ \\
\hline Faktor 1 „Wut“ & $+0,19$ & $<0.05$ & Solidarität & $+0,36$ & $<0.01$ \\
\hline Faktor 2 ,Schock“ & $+0,28$ & $<0.01$ & Hass & $-0,03$ & n.s. \\
\hline \multirow[t]{2}{*}{ Faktor 3 „Angst“ } & $+0,01$ & n.s. & Mitleid & $+0,35$ & $<0.01$ \\
\hline & & & Verachtung & $+0,26$ & $<0.01$ \\
\hline \multicolumn{6}{|c|}{ Leser des Artikels mit humanitärer Rahmung } \\
\hline Legitimierung und ... & Pearson-Korr. & $\mathbf{P}$ & Legitimierung und ... & Pearson-Korr. & $\mathrm{p}$ \\
\hline Faktor 1 „Wut“ & $+0,32$ & $<0.01$ & Solidarität & $+0,33$ & $<0.01$ \\
\hline Faktor 2 „Schock“ & $+0,25$ & $<0.05$ & Hass & $-0,21$ & n.s. \\
\hline \multirow[t]{2}{*}{ Faktor 3 „Angst“ } & $+0,01$ & n.s. & Mitleid & $+0,11$ & n.s. \\
\hline & & & Verachtung & $-0,01$ & n.s. \\
\hline \multicolumn{6}{|c|}{ Leser des Artikels mit politisch-militärischer Rahmung } \\
\hline Legitimierung und ... & Pearson-Korr. & $\mathbf{P}$ & Legitimierung und ... & Pearson-Korr. & $\mathrm{p}$ \\
\hline Faktor 1 „Wut" & $+0,04$ & n.s. & Solidarität & $+0,31$ & $<0.01$ \\
\hline Faktor 2 „Schock“ & $+0,20$ & n.s. & Hass & $+0,05$ & n.s. \\
\hline \multirow[t]{2}{*}{ Faktor 3 „Angst“ } & $+0,01$ & n.s. & Mitleid & $+0,41$ & $<0.01$ \\
\hline & & & Verachtung & $+0,40$ & $<0.01$ \\
\hline
\end{tabular}

Zusammenhänge zwischen Emotionen und Legitimierung je nach Experimentalbedingung unterschiedlich ausfallen. Da sich bei den bisherigen Analysen meist ein Haupteffekt des Bezugsrahmen ergab, haben wir die Korrelationen nur nach Lesern der beiden Artikelversionen aufgeschlüsselt (Tabelle 4): Positive Korrelationen zwischen Wut bzw. Schock sowie Zustimmung zum Bundeswehreinsatz waren nur bei Lesern des Artikels mit humanitärer Rahmung signifikant, aber nicht bei Lesern des Artikels mit politischmilitärischer Perspektive. Solidarität wiederum korrelierte bei Lesern beider Artikelversionen signifikant positiv mit der Akzeptanz des Bundeswehreinsatzes. Dabei ist zu beachten, dass der humanitär argumentierende Beitrag sowohl mehr Solidarität als auch höhere Zustimmung zur Truppenentsendung hervorrief als der politisch-militärische Artikel (vgl. Tabelle 3 und Abbildung 2). Im einen Fall ist die Korrelation daher gleichsam ,auf höherem Niveau', im anderen Fall , auf niedrigerem Niveau'. Dieser Befund lässt sich als gewisser Beleg für unsere theoretischen Überlegungen werten (vgl. Abbildung 1 oben). Anders als erwartet, war Mitleid bei Lesern des humanitär rahmenden Artikels zwar vorhanden, spielte aber offensichtlich keine Rolle für die Akzeptanz des Bundeswehreinsatzes. Bei Lesern des politisch-militärisch argumentierenden Beitrags bestand dagegen ein solcher Zusammenhang. Das gleiche betrifft Verachtung. Allerdings haben wir es hier erneut mit einer Korrelation , auf niedrigem Niveau' zu tun: Denn sowohl das Gefühl der Verachtung als auch die Zustimmung zum Bundeswehreinsatz waren bei Lesern des Artikels mit politisch-militärischer Rahmung vergleichsweise geringer ausgeprägt (vgl. Tabelle 3 und Abbildung 2). 
Tabelle 5: Legitimierung des Bundeswebreinsatzes durch die Probanden (Index) ${ }^{\text {A) }}$ - Zweifaktorielle Varianzanalysen mit ausgewäblten Kovariaten

\begin{tabular}{|c|c|c|c|}
\hline Varianzanalyse 1 „Bundeswebr“ & Partielles Eta ${ }^{2}$ & F-Wert & $\mathrm{p}$ \\
\hline Faktor 1 „Textlicher Bezugsrahmen“ & 0,06 & 7,46 & $<0.01$ \\
\hline Faktor 2 „Nachrichtenbild“ & 0,03 & 3,83 & n.s. \\
\hline Interaktion Faktor $1 *$ Faktor 2 & 0,00 & 0,52 & n.s. \\
\hline Kovariate 1 „Einstellung zur Bundeswehr“ A) & 0,01 & 1,30 & n.s. \\
\hline Kovariate 2 „Vertrauen in Bundeswehr“ B) & 0,06 & 8,40 & $<0.01$ \\
\hline \multicolumn{4}{|l|}{ Gesamt Eta $^{2}=0,18$} \\
\hline Varianzanalyse 2 „Politische Aufgabe BRD“ & Partielles Eta ${ }^{2}$ & F-Wert & $\mathrm{p}$ \\
\hline Faktor 1 „Textlicher Bezugsrahmen“ & 0,06 & 7,72 & $<0.01$ \\
\hline Faktor 2 „Nachrichtenbild“ & 0,04 & 5,47 & $<0.05$ \\
\hline Interaktion Faktor $1 *$ Faktor 2 & 0,00 & 0,03 & n.s. \\
\hline Kovariate „Frieden in der Welt sichern“ C) & 0,12 & 16,74 & $<0.001$ \\
\hline \multicolumn{4}{|l|}{ Gesamt $\mathrm{Eta}^{2}=0,21$} \\
\hline $\begin{array}{l}\text { n=130 Probanden. } \\
\text { A) Skala von }+2 \text { („Positiv“) bis }-2 \text { („Negativ“). } \\
\text { B) Skala von } 1 \text { („Überhaupt kein Vertrauen“) bis } 5 \text { (, } \\
\text { wehr“ und Legitimierungs-Index: Pearsons r }=+0,29 \\
\text { C) Skala von } 1 \text { („Überhaupt nicht wichtig“) bis } 5 \text { („Seh } \\
\text { Legitimierungs-Index: Pearsons } r=+0,36 \text { (p<0.01). }\end{array}$ & $\begin{array}{l}\text { s Vertrauen“). K } \\
\text { ig“). Korrelation }\end{array}$ & lation „Ve & sichern" und \\
\hline
\end{tabular}

Abschließend sind Drittvariablen zu diskutieren. Wir haben mehrere zweifaktorielle Varianzanalysen mit jeweils anderen Drittvariablen durchgeführt. Diese zeigten nur in zwei Fällen signifikante intervenierende Effekte (Tabelle 5). Während die Voreinstellung der Probanden zur Bundeswehr keinen Einfluss auf ihre Legitimierung des konkreten Einsatzes in Mlandi hatte, spielte das Vertrauen in die Bundeswehr sehr wohl eine Rolle: Je höher ihr generelles Vertrauen in die Bundeswehr, desto eher stimmten die Probanden dem angeblichen Einsatz in Mlandi zu (Pearsons $\mathrm{r}=+0,29 ; \mathrm{p}<0.01$ ). Der Anteil erklärter Varianz war vergleichbar mit jenem des ersten Experimentalfaktors (Varianzanalyse 1 in Tabelle 5). Eine größere Bedeutung hatte die Überzeugung der Probanden, dass Friedenssicherung eine wichtige Aufgabe der deutschen Politik sei (Varianzanalyse 2): Diese Ansicht korrelierte noch stärker mit der Legitimierung des Einsatzes in Mlandi (Pearsons $r=+0,36 ; p<0.01)$. Da die Einstellung zur Friedenssicherung gegen Ende des Fragebogens und damit nach dem Treatment abgefragt wurde, können wir jedoch bei diesem ,stimulusnahen' Merkmal nicht ganz ausschließen, dass die Angaben der Probanden teilweise auch durch das Treatment beeinflusst wurden. Die anderen Drittvariablen spielten keine nennenswerte Rolle, was bei anderen Politikfeldern allerdings durchaus der Fall sein kann. Warum nur bei den genannten Drittvariablen erkennbare Effekte auftraten, kann unterschiedliche Gründe haben, die sich nicht zweifelsfrei klären lassen. Dass das Vertrauen in die Bundeswehr, nicht aber die Einstellung zur Truppe eine Rolle spielte, könnte damit zusammenhängen, dass die Vertrauensfrage das Einstellungskonstrukt valider erfasst als die direkte Einstellungsfrage. Eine naheliegende Erklärung wäre auch, dass spezifisch themengebundene Drittvariablen wie das Vertrauen in die Bundeswehr eine stärkere Bedeutung bei dem Thema hatten als etwa generelle Politikverdrossenheit. Diese wiederum könnte bei innenpolitischen Themen stärker durchschlagen. 


\section{Schlussfolgerungen}

Die vorliegende Studie untersuchte den Einfluss der Politikberichterstattung auf die Legitimierung politischer Entscheidungen durch die Bürger und dabei auch die Rolle von Emotionen. Neben themenrelevanten Voreinstellungen der Probanden können offensichtlich auch der Bezugsrahmen, in den die Medien ein Problem und die Entscheidung zur Problemlösung stellen, teilweise aber auch Nachrichtenbilder die Zustimmung der Rezipienten zur politischen Entscheidung beeinflussen. Dahinter können zwei Wirkungsmechanismen stehen: Einerseits kann ein Bezugsrahmen bestimmte Aspekte (z. B. Humanität) kognitiv salienter machen, was den Stellenwert der daran geknüpften Bewertungen (z. B. positiver Affekt) für die Legitimierung der Entscheidung erhöht. Andererseits kann ein Bezugsrahmen auch Emotionen (z. B. Solidarität) hervorrufen, die an bestimmte Grundwerte (z. B. Humanität) appellieren. Wenn die inhaltliche Rahmung dann eine Entscheidung (implizit) als förderlich für diese Grundwerte vermittelt, erhöht das die Zustimmung der Bürger. Die Korrelationen zwischen Emotionen und Legitimierung der Probanden deuten darauf hin, dass dieser Mechanismus im Experiment zumindest teilweise wirksam wurde.

Damit scheinen Emotionen bei bestimmten politischen Fragen durchaus eine wichtige Rolle zu spielen. Ob und bei welchen Politikfeldern das ebenfalls so ist, können wir aus unseren Befunden nicht ableiten. Denkbar ist das aber beispielsweise - schon aufgrund des hohen Involvement vieler Bürger - bei Arbeitsmarkt- und Sozialreformen. Und auch die Entscheidung für politische Kandidaten hängt sicherlich nicht nur von deren Kompetenz und Vertrauenswürdigkeit ab, sondern auch von Gefühlen wie Ärger oder Sympathie, die ihnen die Wähler entgegenbringen. Einen Hinweis in diese Richtung gibt z. B. die Bundestagswahl 2002. Raschke (2003) hat in diesem Zusammenhang unterschiedliche Mehrheiten in der deutschen Bevölkerung ausgemacht. Die Union und ihr Kandidat Stoiber hätten damals zwar die ökonomische Mehrheit auf ihrer Seite gehabt. Allerdings habe dann u. a. die Flut in Ostdeutschland das Moment der Solidarität auf die Agenda gebracht, das Schröder weit besser bediente als sein Herausforderer.

Auch wenn sich die zentralen Hypothesen unseres Experiments weitgehend bestätigten, unterliegt unsere Studie einigen - teils methodischen, teils inhaltlichen - Beschränkungen:

Experimente dienen oft dazu, grundsätzliche Wirkungsmechanismen (z. B. ThirdPerson-Effekt) nachzuweisen. Bei einer Fragestellung zu politischer Kommunikation liegt dagegen zunächst eine Feldstudie nahe. Im vorliegenden Fall würde man eine Inhaltsanalyse der Berichterstattung über Auslandseinsätze der Bundeswehr mit einer Bevölkerungsbefragung kombinieren. Allerdings kann man im Querschnitt nur Korrelationen feststellen und keine Kausalaussagen treffen. Im Längsschnitt wäre das möglich, aber dafür müsste man die Berichterstattung und die Bevölkerungsansichten zu mehreren Zeitpunkten erheben, was im zweiten Fall ex post nicht möglich ist. Ein Experiment war daher für unsere Fragestellung angemessen - zumal sich auf diese Weise empirisch bedeutsame Berichterstattungsmuster gezielt manipulieren ließen.

Man mag einwenden, dass die einmalige Stimulusgabe eine kumulative, konsonante Berichterstattung nicht hinreichend repräsentiert (vgl. Scheufele 2004) und daher - gerade was die Emotionen der Probanden angeht - nur kurzfristige Effekte erfasst wurden. Der Einwand lässt sich aber teilweise entkräften: Denn die empirisch begründete Artikelversion mit humanitärer Rahmung dürfte jene Ansichten aktualisiert haben, welche die Probanden in den letzten Jahren aufgrund der Berichterstattung über tatsächliche Bundeswehreinsätze (z. B. im Kosovo) herausgebildet haben. Die Effekte bei Lesern 
des humanitär argumentierenden Artikels gehen dann nicht nur auf das experimentelle Treatment zurück, sondern auch auf diese frühere Berichterstattung. Allerdings ist trotz signifikanter Haupteffekte nicht von der Hand zu weisen, dass die Erklärungskraft der Varianzanalysen $\left(\mathrm{Eta}^{2}\right)$ nicht extrem hoch ausgefallen ist. Das mag vor allem damit zusammenhängen, dass wir die Effekte experimentell und nicht im Feld gemessen haben.

\begin{tabular}{lll} 
Tabelle 6: $\begin{array}{l}\text { Legitimierung durch Politikberichterstattung (modifiziert nach Schenfele } \\
\text { 2005a) }\end{array}$ & \\
\hline Ebene & Aspekt & Medieneinfluss (Auswahl) \\
\hline Begründung & Problemdefinition & $\begin{array}{l}\text { Mediales Forum für die Problemdefinition (Bezugsrabmen, } \\
\text { Rollenzuschreibung usw.) der Entscheidungsträger oder } \\
\text { ihrer Kritiker }\end{array}$ \\
& $\begin{array}{l}\text { Mediales Forum für Pro-/Contra-Argumente zur Problem- } \\
\text { lösung }\end{array}$ \\
Innerparteilich & $\begin{array}{l}\text { Mediendarstellung eines innerparteilichen Konsenses/Dis- } \\
\text { senses }\end{array}$ \\
\hline Zustimmung Parlamentarisch & $\begin{array}{l}\text { Mediendarstellung eines parlamentarischen Konsenses/Dis- } \\
\text { senses }\end{array}$ \\
Gesellschaftlich & $\begin{array}{l}\text { Mediendarstellung einer gesellschaftlichen Zustimmung/Ab- } \\
\text { lehnung }\end{array}$ \\
\hline
\end{tabular}

Unsere theoretischen Überlegungen haben wir am exemplarischen Politikfeld der Außen-bzw. Sicherbeitspolitik überprüft. Bei anderen Themen können die Befunde möglicherweise stärker oder schwächer ausfallen bzw. durch andere Drittvariablen moderiert werden als im vorliegenden Fall (vgl. Abschnitt 5). Das exemplarische Thema eignete sich aber für unsere Fragestellung, weil Bundeswehreinsätze außerhalb des Erfahrungsbereichs der meisten Menschen liegen und Medieneinflüsse damit wahrscheinlicher waren als bei anderen Themen. Darüber hinaus sind der inhaltliche Bezugsrahmen und das Nachrichtenbild nur zwei Merkmale, die sich auf die Zustimmung der Bürger zu politischen Entscheidungen auswirken können. Scheufele (2005a, 2007) hat mehrere Aspekte auf der Begründungs- und der Zustimmungsebene politische Legitimität diskutiert - ohne jedoch die Wirkungsfrage zu klären (Tabelle 6). Auf der Begründungsebene können Medien eine politische Entscheidung stützen, indem sie der Problemdefinition der Entscheidungsträger ein Forum bieten, oder sie schwächen, wenn sie die Sichtweise der Kritiker hochspielen. Der im Experiment manipulierte Bezugsrahmen gehört zur Problemdefinition. Medien können eine Entscheidung aber auch stützen oder schwächen, indem sie Argumente für oder gegen die Problemlösung, also die Entscheidung selbst vorbringen. Vergleichbares beschreibt die Theorie instrumenteller Aktualisierung (Kepplinger et al. 1989). Oder Medien thematisieren das Privatleben eines Entscheidungsträgers und desavouieren damit dessen Ansprüche auf ein Parteiamt oder politische Entscheidungen, für die er steht (vgl. Scheufele, 2007). Auf der Zustimmungsebene geht es dagegen um innerparteiliche Zustimmung, parlamentarische Mehrheiten und gesamtgesellschaftliche Unterstützung für die Entscheidung. Berichten Medien z. B. über innerparteiliche Kritiker oder eine drohende Abstimmungsniederlage, dann delegitimieren sie eine Entscheidung.

Allerdings ist jede Mediendarstellung, wie schon eingangs betont wurde, auch eine Funktion der politischen Realität. Welchen Anteil diese an der Mediendarstellung politischer Entscheidungen hat, kann nur empirisch geklärt werden. Das gilt auch für die 
Frage, ob das mediale Bild einer Entscheidung im Hinblick auf die erwähnten Begründungs- und Zustimmungsaspekte politischer Legitimität angemessen ist. Unabhängig von der Antwort auf beide Fragen sind die nachgewiesenen Effekte aber Medienwirkungen. Idealtypisch sind dabei zwei Phänomene zu unterscheiden: (1) Transportieren die Medien - im Sinne einer ,Vermittlerrolle - die Bezugsrahmen, Argumente oder Sichtweisen der politischen Entscheidungsträger, dann handelt es sich bei der Zustimmung bzw. Ablehnung der Bürger um eine Wirkung der Handlungen, Statements oder Beschlüsse von Politikern. Diese ist aber - gerade bei Themen außerhalb des eigenen Erfahrungshorizonts der Bürger - ohne die Medien nicht denkbar. Hier könnte man von einer medienvermittelten Wirkung der Politik sprechen. (2) Konstruieren die Medien dagegen - im Sinne einer ,prägenden Rolle ${ }^{`}$ - das öffentliche Bild von politischen Akteuren und deren Entscheidungen, dann handelt es sich bei der Legitimierung seitens der Bürger um Medienwirkungen. Diese sind aber ohne die Handlungen, Statements oder Beschlüsse von Politikern nicht denkbar. Hier könnte man von einer politikinduzierten Wirkung der Medien sprechen. In der Realität greifen beide Phänomene ineinander. Abschließend sei nochmals betont, dass wir nur einen Aspekt politischer Legitimität bzw. Legitimierung betrachtet haben - nämlich die Zustimmung zu konkreten Outputs des politischen Systems, also „instrumental support“ (Easton 1975). Die Zustimmung zu politischen Akteuren oder die langfristige Akzeptanz des politischen Systems generell dürften von der Legitimierung der konkreten Outputs allerdings nicht unberührt bleiben (vgl. z. B. Easton 1975, 1979; Greiffenhagen 1997; Westle 1989, 1990).

\section{Literaturverzeichnis}

Akademie der Bundeswehr für Information und Kommunikation AIK (2001): Demoskopisches Meinungsbild in Deutschland zur Sicherheits- und Verteidigungspolitik 2000. AIK-Texte 1/2001. Strausberg.

Bentele, Günter (1992): Fernsehen und Realität. Ansätze zu einer rekonstruktiven Medientheorie. In: Hickethier, Knut/Schneider, Irmela (Hrsg.), Fernsehtheorien. Dokumentation der GFFTagung 1990. Berlin, S. 45-67.

Bierhoff, Hans Werner/Montada, Leo (Hrsg.) (1988): Altruismus. Bedingungen der Hilfsbereitschaft. Göttingen.

Brosius, Hans-Bernd (1995): Alltagsrationalität in der Nachrichtenrezeption. Ein Modell der Wahrnehmung und Verarbeitung von Nachrichteninhalten. Opladen.

Easton, David (1975): A Re-Assessment of the Concept of Political Support. In: British Journal of Political Science 5, S. 453-457.

Easton, David (1979): A systems analysis of political life. New York et al.

Eilders, Christiane/Lüter, Albrecht (2000): Germany at War. Competing Framing Strategies in German Public Discourse. In: European Journal of Communication 15 (Special Issue): The Media and the Kosovo Conflict; hrsg. von Golding, Peter/McQuail, Denis, S. 415-428.

Früh, Hannah/Fahr, Andreas (2006): Erlebte Emotionen. Messung von Rezeptionsemotionen am Beispiel legitimierter Gewalt im Spielfilm. In: Publizistik 51, S. 24-38.

Fuchs, Dieter (1987): Trends politischer Unterstützung in der Bundesrepublik. In: Berg-Schlosser, Dirk/Schissler, Jakob (Hrsg.), Politische Kultur in Deutschland. Bilanz und Perspektiven der Forschung (Politische Vierteljahresschrift, Sonderheft 18). Opladen, S. 357-377.

Greiffenhagen, Martin (1997): Politische Legitimität in Deutschland. Gütersloh.

Hatfield, Elaine/Cacioppo, John T./Rapson, Richard L. (1994): Emotional Contagion. Cambridge.

Heller, Hermann (1983): Staatslehre. 6. Auflage, Tübingen.

Iyengar, Shanto/Simon, Adam (1993): News Coverage of the Gulf Crisis and Public Opinion. A Study of Agenda Setting, Priming and Framing. In: Communication Research 20, S. 365-383. 
Jacobs, Jörg (1999): Einstellungen zur politischen Ordnung in Transformationsländern. Lebenssituation und zentrale Einstellungen als Determinanten in den Neuen Bundesländern, Polen, Tschechien und Ungarn. Arbeitsberichte - Discussion Papers Nr. 14/1999. Frankfurter Institut für Transformationsstudien. Frankfurt am Main.

Jarren, Otfried/Donges, Patrick (2002a): Politische Kommunikation in der Mediengesellschaft. Eine Einführung. Band 1: Verständnis, Rahmen und Strukturen. Wiesbaden.

Jarren, Otfried/Donges, Patrick (2002b): Politische Kommunikation in der Mediengesellschaft. Eine Einführung. Band 2: Akteure, Prozesse und Inhalte. Wiesbaden.

Jarren, Otfried/Donges, Patrick/Weßler, Hartmut (1996): Medien und politischer Prozess. Eine Einleitung. In: Jarren, Otfried/Schatz, Heribert/Weßler, Hartmut (Hrsg.), Medien und politischer Prozess. Politische Öffentlichkeit und massenmediale Politikvermittlung im Wandel. Opladen, S. 9-40

Jertz, Walter (2001): Krieg der Worte - Macht der Bilder. Manipulation oder Wahrheit im KosovoKonflikt. Bonn.

Kepplinger, Hans Mathias (1998): Die Demontage der Politik in der Informationsgesellschaft. Freiburg, München.

Kepplinger, Hans Mathias (2001): Der Ereignisbegriff in der Publizistikwissenschaft. In: Publizistik 46, S. 117-139.

Kepplinger, Hans Mathias/Brosius, Hans-Bernd/Staab, Joachim Friedrich/Linke, Günter (1989): Instrumentelle Aktualisierung. Grundlagen einer Theorie publizistischer Konflikte. In: Kaase, Max/Schulz, Winfried (Hrsg.), Massenkommunikation. Theorien, Methoden, Befunde (Kölner Zeitschrift für Soziologie und Sozialpsychologie; Sonderheft 30). Opladen, S. 199-220.

Knieper, Thomas/Müller, Marion G. (Hrsg.) (2005): War Visions. Bildkommunikation und Krieg. Köln.

Kroeber-Riehl, Werner (1996): Konsumentenverhalten. 6. Auflage, München.

Löffelholz, Martin (2004): Kriegs- und Krisenkommunikation als Forschungsfeld. In: Löffelholz, Martin (Hrsg.), Krieg als Medienereignis 2. Krisenkommunikation im 21. Jahrhundert. Wiesbaden, S. 13-55.

Mangold, Roland/Unz, Dagmar/Winterhoff-Spurk, Peter (2001):Zur Erklärung emotionaler Medienwirkungen. Leistungsfähigkeit, empirische Überprüfung und Fortentwicklung theoretischer Ansätze. In: Rössler, Patrick/Hasebrink, Uwe/Jäckel, Michael (Hrsg.), Theoretische Perspektiven der Rezeptionsforschung (Angewandte Medienforschung, Bd. 17). München, S. 163-180.

Manstead, Antony S. R./Fischer, Agneta H. (2001): Social Appraisal: The Social World as Object of and Influence on Appraisal Processes. In: Scherer, Klaus R./Schorr, Angela/Johnstone, T. (Hrsg.), Appraisal Processes in Emotion. Theory, Methods, Research. Oxford, S. 221-232.

Maurer, Marcus (2003): Politikverdrossenheit durch Medienberichte. Eine Paneluntersuchung. Konstanz.

Meder, Gerhard (1998): Zur Neubestimmung der Rolle der Bundeswehr in den deutschen Printmedien. In: Kempf, Wilhelm/Schmidt-Regener, Irena (Hrsg.), Krieg, Nationalismus, Rassismus und die Medien. Münster, S. 201-210.

Merten, Jörg/Krause, Rainer (1993): DAS (Differentielle Affekt Skala). Arbeiten der Fachrichtung Psychologie, Universität des Saarlandes, Nr. 173. Saarbrücken. In: http://chi.psychologie.uni mannheim.de/psycho3/lehrealt/ss00/medgef/medgefneu.htm [19.11.2005].

Nelson, Thomas E./Oxley, Zoe M./Clawson, Rosalee A. (1997): Towards a Psychology of Framing Effects. In: Political Behaviour 19, S. 221-246.

Ortony, Andrew/Clore, Gerald L./Collins, Allan (1988): The Cognitive Structure of Emotion. Cambridge.

Price, Vincent/Tewksbury, David/Powers, Elisabeth (1997): Switching trains of thought: The Impact of News Frames on Readers' Cognitive Responses. In: Communication Research 24, S. 481-506.

Rabehl, Thomas (Hrsg.) (2000): Das Kriegsgeschehen 1999. Daten und Tendenzen der Kriege und bewaffneten Konflikte. Arbeitsgemeinschaft Kriegsursachenforschung. Opladen.

Raschke, Joachim (2003): Zwei Lager, drei Mehrheiten und der regierende Zufall. Zur Einordnung der Bundestagswahl 2002. In: Forschungsjournal NSB 16, S. 14-24. 
Reeb, Hans-Joachim (2004): Öffentlichkeit als Teil des Schlachtfeldes. Grundlagen der Kriegskommunikation aus militärischer Perspektive. In: Löffelholz, Martin (Hrsg.), Krieg als Medienereignis 2. Krisenkommunikation im 21. Jahrhundert. Wiesbaden, S. 197-213.

Robinson, Piers (2000a): The News Media and Intervention. Triggering the Use of Air Power During Humanitarian Crisis. In: European Journal of Communication 15 (Special Issue): The Media and the Kosovo Conflict; hrsg. von Golding, Peter/McQuail, Denis, S. 405-414.

Robinson, Piers (2000b): The Policy-Media Interaction Model. Measuring Media Power During Humanitarian Crisis. In: Journal of Peace Research 37/5, S. 613-633.

Rosenberg, Morris J. (1956): Cognitive Structure and Attitudinal Affect. In: Journal of Abnormal and Social Psychology 53, S. 367-372.

Rucht, Dieter (1991): Parteien, Verbände und Bewegungen als Systeme politischer Interessenvermittlung. Wissenschaftszentrum für Sozialforschung Berlin. WZB Discussionpaper FS III, S. 91-101. Berlin.

Sarcinelli, Ulrich (1998a): Legitimität. In: Jarren, Otfried/Sarcinelli, Ulrich/Saxer, Ulrich (Hrsg.), Politische Kommunikation in der demokratischen Gesellschaft. Ein Handbuch mit Lexikonteil. Opladen, S. 254-267.

Sarcinelli, Ulrich (Hrsg.) (1998b): Politikvermittlung und Demokratie in der Mediengesellschaft. Beiträge zur politischen Kommunikationskultur. Opladen.

Sargent, Stephanie/Zillmann, Dolf (1999): Image Effects on Selective Exposure to News Stories. Paper presented at the 49th annual meeting of the International Communication Association, ICA in San Francisco/USA, May 23-31.

Schenk, Michael (2002): Medienwirkungsforschung. 2., vollständig überarbeitete Auflage. Tübingen.

Scherer, Klaus R. (1998): Emotionsprozesse im Medienkontext: Forschungsillustrationen und Zukunftsperspektiven. In: Medienpsychologie 10, S. 276-293.

Scherer, Klaus R. (2001): Appraisal Considered as a Process of Multi-Level Sequential Checking. In: Scherer, Klaus R./Schorr, Angela/Johnstone, Tom (Hrsg.), Appraisal Processes in Emotion. Theory, Methods, Research. New York, S. 92-120.

Scheufele, Bertram (2001): Visuelles Medien-Framing und Framing-Effekte. Zur Analyse visueller Kommunikation aus der Framing-Perspektive. In: Knieper, Thomas/Müller, Marion G. (Hrsg.), Kommunikation visuell - Das Bild als Forschungsgegenstand - Grundlagen und Perspektiven. Köln, S. 144-158.

Scheufele, Bertram (2003): Frames - Framing - Framing-Effekte. Theoretische und methodische Grundlegung sowie empirische Befunde zur Nachrichtenproduktion. Wiesbaden.

Scheufele, Bertram (2004): Framing-Effekte auf dem Prüfstand. Eine theoretische, methodische und empirische Auseinandersetzung mit der Wirkungsperspektive des Framing-Ansatzes. In: Medien \& Kommunikationswissenschaft 52/1, S. 30-55.

Scheufele, Bertram (2005a): Mediale Legitimierung von Kriegen durch Rollen-Zuschreibung. Eine explorative Studie zur Berichterstattung deutscher Nachrichtenmagazine über den KosovoKrieg. In: Medien \& Kommunikationswissenschaft 53/2-3, S. 352-368.

Scheufele, Bertram (2005b): Sexueller Missbrauch - Mediendarstellung und Medienwirkung. Wiesbaden.

Scheufele, Bertram (2007): Formen und Effekte medialer Legitimierung von Politik. Theoretische Überlegungen und empirische Befunde. Unveröffentlichtes Manuskript. Jena.

Schwab-Trapp, Michael (2000): Der deutsche Diskurs über den Jugoslawienkrieg. In: Grewenig, Adi/Jäger, Margret (Hrsg.), Medien in Konflikten. Holocaust - Krieg - Ausgrenzung. Duisburg, S. 97-100.

Schwab-Trapp, Michael (2002): Kriegs-Diskurse. Die politische Kultur des Krieges im Wandel 1991-1999. Opladen.

Steinkamm, Armin A. (2000): Völkerrecht, Humanitäre Intervention und Legitimation des Bundeswehr-Einsatzes. Völker- und wehrrechtliche Aspekte des Kosovo-Konflikts 1999. In: Bayerische Landeszentrale für politische Bildungsarbeit (Hrsg.), Der Kosovo-Konflikt. Ursachen - Akteure - Verlauf. München, S. 335-362.

Szukala, Andrea (2003): Medien und öffentliche Meinung im Irakkrieg. In: Aus Politik und Zeitgeschichte B 24-25/2003, S. 5-34. 
Tenscher, Jens (2003): Professionalisierung von Politikvermittlung? Politikvermittlungsexperten im Spannungsfeld von Politik und Massenmedien. Wiesbaden.

Ulich, Dieter/Mayring, Philipp (2003): Psychologie der Emotionen. Stuttgart.

Weber, Max (1972): Wirtschaft und Gesellschaft. Studienausgabe. 5. Auflage. Tübingen.

Westle, Bettina (1989): Politische Legitimität. Theorien, Konzepte, empirische Befunde. BadenBaden.

Westle, Bettina (1990): Legitimität der Parteien und des Parteiensystems in der Bundesrepublik Deutschland. In: Kölner Zeitschrift für Soziologie und Sozialpsychologie 42, S. 401-427.

Wirth, Werner/Stiehler, Hans-Jörg/Wünsch, Carsten (Hrsg.) (2007): Dynamisch-transaktional denken. Theorie und Empirie der Kommunikationswissenschaft. Für Werner Früh. Köln.

Wolling, Jens (1999): Politikverdrossenheit durch Massenmedien? Der Einfluss der Medien auf die Einstellungen der Bürger zur Politik. Opladen.

Zillmann, Dolf (2004): Emotionspsychologische Grundlagen. In: Mangold, Roland/Vorderer, Peter/Bente, Gary (Hrsg.), Lehrbuch der Medienpsychologie. Göttingen et al., S. 101-128. 